\title{
Assessment of Rapid Diagnostic Tests Algorithms in Transfusion Medicine Setting
}

\author{
Modibo Coulibaly1, Bakary Maiga'2,3, Dramane Samaké4, Moussa Diawara1, Mahamadou Traoré1, \\ Valentin Sagara1, Bréhima Traoré5, Oumar Guindo5, Amagana Dolo6
}

${ }^{1}$ Department of Biomedical Laboratory, Sominé DOLO Hospital, Mopti, Mali

${ }^{2}$ Department of Research and Training, National Center for Transfusion, Bamako, Mali

${ }^{3}$ Faculty of Medicine and Odonto-Stomatology, University of Sciences, Techniques and Technologies, Bamako, Mali

${ }^{4}$ Department of Medicine, Sominé DOLO Hospital, Mopti, Mali

${ }^{5}$ Department of Public Health, Sominé DOLO Hospital, Mopti, Mali

${ }^{6}$ Faculty of Pharmacy, University of Sciences, Techniques and Technologies, Bamako, Mali

Email: modibocoulibaly@yahoo.com

How to cite this paper: Coulibaly, M. Maiga, B., Samaké, D., Diawara, M., Traoré, M., Sagara, V., Traoré, B., Guindo, O. and Dolo, A. (2021) Assessment of Rapid Diagnostic Tests Algorithms in Transfusion Medicine Setting. Advances in Biological Chemistry, 11, 52-63. https://doi.org/10.4236/abc.2021.111005

Received: January 19, 2021

Accepted: February 22, 2021

Published: February 25, 2021

Copyright () 2021 by author(s) and Scientific Research Publishing Inc. This work is licensed under the Creative Commons Attribution International License (CC BY 4.0).

http://creativecommons.org/licenses/by/4.0/

\section{(c) (i) Open Access}

\begin{abstract}
Background: The rapid diagnostic tests play a pivotal role in the screening of viral markers in blood qualification for transfusion in limited resource setting. Therefore, it is important to assess their analytical performances to ensure their proper functioning. Material and Methods: We performed a crosssectional study by successive recruitment to assess the diagnostic value of rapid diagnostic tests algorithms using ELISA as a reference test. A total of 661 blood from donors were enrolled for this study. Rapid Diagnostic Tests (RDTs) and ELISA tests were performed for each sample by a couple of double-blinded biotechnologists. Data were collected on case report form and captured in Microsoft Excel then the file was imported and analyzed using $\mathrm{R}$ software version 4.0.3. Results: The diagnostic accuracy for the algorithms are summarized in Table 1 . For HIV-algorithm, the internal validity parameters were as follow: sensitivity (sens) $99.0 \%$ (95\% CI $=97.8,99.5)$; specificity (spec) $98.3 \%$ (95\% CI = 90.9, 99.7); positive likelihood ratio (PLR) 57.4 (95\% $\mathrm{CI}=8.2,401.0)$; negative likelihood ratio (NLR) 0.01 (95\% CI = 0.0005, 0.02); diagnostic odd ratio (DOR) 4710. HBV-Ag/Ab RDTs achieve the following diagnostic accuracy: sens $99.7 \%(95 \% \mathrm{CI}=98.3,99.9)$; spec $98.8 \%(95 \% \mathrm{CI}=$ 96.9, 95.5); PLR 81.8 (95\% CI = 30.9, 217.0); NLR 0.003 (95\% CI = 0.0004, 0.02); DOR 14,110. The analytical performances of HCV-Ab RDTs were as follow: sens $98.7 \%(95 \% \mathrm{CI}=97.5,99.4)$; spec $93.1 \%(95 \% \mathrm{CI}=78.0,98.1)$; PLR 14.3 (95\% CI = 3.8, 54.5); NLR 1.5 (95\% CI = 0.8, 2.8); DOR 962.6. The parameters evaluating the external validity of RDTs screening for the three viral markers when the theorical prevalence was $<5 \%$ are summarized in
\end{abstract}


Figure 3. At the prevalence $<5 \%$, the NPV of the three RDTs were $99.96 \%$, 99.99\% and $99.94 \%$. At the same prevalence, we found the following Positive Predictive Values (PPV) 70.82\%, 77.59\% and 37.35\% for HIV-Ag/Ab RDTs, HBV-Ag RDTs and HCV-Ab RDTs algorithms, respectively. The overall areas under the received operating characteristic (ROC) curves were 98.6\%, 99.2\% and 99.2\%; 95.9\% for HIV-Ag/Ab RDTs, HBV-Ag RDTs and HCV-Ab RDTs algorithms, respectively. Conclusion: RDTs algorithms can play a pivotal role in the screening of HIV-Ab/Ag, HBs-Ag in the setting of resources limited-countries where financial and technical expertise shortages are a standard fare. However, their use for diagnostic purposes must be done with great caution and the result must necessarily be confirmed with an ELISA or molecular technique particularly for HCV-RDTs algorithm which achieved an NLR value $>0.1$.

\section{Keywords}

Rapid Diagnostic Tests, Analytical Performances, Assessment, Transfusion Medicine

\section{Introduction}

Screening for transfusion-transmissible infections (TTI) is mandatory in any national blood transfusion program. However, it should be noted that there are no screening programs for TTI which has no limits, the exclusive safety, perceived as the formal absence of infectious risk would be difficult to reach [1]. Nevertheless, much effort has been put into developing strategies to reduce the risk of transmission of infectious agents through blood transfusion. There is a great deal of variability in screening strategies and in the choice of tests. In addition, the tests used have different sensitivities and specificities. The specificity increasing from immunochromatographic tests called rapid diagnostic tests (RDTs) to so-called molecular tests. As a result, screening strategies may differ from high-income countries to low-income countries and between different levels within the health system of the same country [2]. Despite the combination of sensitive RDTs and specific tests like ELISA, studies have shown that there is a residual risk of TTI [3] [4] [5]. This residual risk is even higher in low-income countries than in high-income countries. The molecular tests can detect viral DNA or RNA and are effective in detecting infected donors who are in a period of seroconversion [6]. However, the implementation of these tests in routine clinical laboratories encounters technical and financial difficulties due to the lack of qualified persons and the cost of equipment and infrastructure particularly in low-income countries and especially in the provinces [4]. The RDTs which are simple in use and do not need high qualified biologists, heavy equipment's and infrastructures play a pivotal role and represent an alternative way for blood screening in low-income setting [7]. The analytical performance of RDTs are 
sometimes could be altered by poor storage, transport conditions over long distance and manufacturing defect upstream the clinical laboratory [8] [9]. The aim of this work is to assess the diagnostic value of HIV, HBV and HCV RDTs algorithms by using the ELISA as a reference test.

\section{Materials and Methods}

We carried out a cross-sectional assessment of RDTs algorithms analytical performances by using blood from donors from December 2016 to June 2017. The sample size was computed according to the prevalence of HBV among blood donors in our hospital (8.9\%) from our previous study. By setting the precision to $5 \%$ and the $95 \%$ confidence interval (CI) to, the number of blood donors required was 661 . Blood donors were selected after a medical check and were presumed to be free from apparent pathologies. Samples were taken by venipuncture at the elbow fold with the vacutainer system. About $4 \mathrm{ml}$ of whole blood were collected for each donor on a dry tube with a coagulation activator and on a tube with EDTA. For HIV serology, we used the WHO-recommended testing strategy when the prevalence is low $(<5 \%)$. This strategy was based on the use of 3 TDRs Alere ${ }^{\mathrm{TM}}$ Determine HIV-1/2 Ag/Ab Combo Dipstick (Alere medical Co., Ltd., Chiba, Japan), SD Bioline HIV-1/2 3.0 Card (Standard Diagnostic, Inc, Gyeonggi, Korea) and the First Response HIV Card 1 - 2.0 (PMC Medical Pvt Ltd, India). For the HBV serology, we used double RDTs: Alere ${ }^{\mathrm{TM}}$ Determine HBsAg Dipstick (Alere medical Co., Ltd., Chiba, Japan) and Cypress HBs Ag Card (Cypress Diagnostics, Langdorp, Belgium). For HCV, we used SD Bioline HCV card (Standard Diagnostic, Inc, Gyeonggi, Korea) and Cypress anti-HCV Card (Cypress Diagnostics, Langdorp, Belgium). The Genscreen ${ }^{\text {TM }}$ ULTRA HIV Ag-Ab (Bio-Rad, Marnes-la-Coquette-France), Monolisa ${ }^{\mathrm{TM}}$ HBs Ag ULTRA (Bio-Rad, Marnes-la-Coquette-France) and Monolisa ${ }^{\mathrm{TM}}$ HCV Ag-Ab ULTRA (Bio-Rad, Marnes-la-Coquette-France) were used as standard tests to evaluate the analytical performances of combined RDTs. Rapid Diagnostic Tests (RDTs) and ELISA tests were performed for each sample by a couple of double-blinded biotechnologists. Data were collected on case report form and captured in Microsoft Excel then the file was imported and analyzed using $\mathrm{R}$ software version 4.0.3. Pearson's chi-square or Fisher's exact test was used for the comparison of proportions. Kolmogorov-Smirnow test was used to evaluate quantitative data normality. Student's $t$-test was used for normal distributed parameters, otherwise Mann-Whitney $U$-test was performed to compare quantitative parameters. The analytical performances of the RDTs and their 95\% confidence intervals (95\% CI) were computed by using the R package DiagnosisMed. ROC curve and nomograms were fitted by the R package ROCR. External validity of RDTs and the plots of positive predictive values (PPV) and negative predictive values (NPV) against theorical prevalence was performed by using the packages risky R. Univariate and multivariate logistic regression were used to evaluate the impact of sociodemographic data on the results of RDTs. The threshold for statistical significance was set at $\mathrm{p} \leq 5 \%$. 


\section{Results}

A total of 661 samples from blood donors were enrolled in this study. Out of the them, $555(84.0 \%)$ were male and 106 (16.0\%) female. The sex-ratio was 5.2. Occasional donors represented $443(67.0 \%)$ versus 218 (33.0\%) of regular donors. The age group from 19 to 29 years was the most represented with $37.8 \%$ followed by those aged from 30 to 39 years (33.9\%) and those aged from 40 years and over (26.8\%). Donors aged 18 were less represented $1.5 \%$. We did not observe a significant difference between the median of age of the donors according to HIV serology statute which were 33.4 vs 32.4 years; $\mathrm{p}=0.42$ for those with negative test and those with positive test, respectively (Figure 1). In contrast, the HBV positive donors were significantly younger than those with negative test 32.5 vs 34.2 years; $\mathrm{p}=0.02$ (Figure 1 ). Likewise, the median age of regular donors was significantly lower than occasional donors 32.03 vs 34.0 years; $p=0.01$ (Figure 1). We did not find a significant difference between the median ages of $\mathrm{HCV}$ positive and negative donors 33.4 vs 28.7 years; $\mathrm{p}=0.22$ (Figure 1 ). The diagnostic accuracy for the three RTDs algorithm are summarized in Table 1. For HIV-Ag/Ab RDTs algorithm analytical performances were as follow: Sensitivity (sens) 99.0\% (95\% CI = 97.8, 99.5); Specificity (spec) $98.3 \%$ (95\% CI = 90.9, 99.7); positive likelihood ratio (PLR) 57.4 (95\% CI = 8.2, 401.0); Negative Likelihood Ratio (NLR) 0.01 (95\% CI = 0.0005, 0.02); diagnostic odd ratio (DOR)

Table 1. Diagnostic performances of the RDTs algorithms and their $95 \%$ confidence interval.

\begin{tabular}{cccc}
\hline \multirow{2}{*}{$\begin{array}{c}\text { Analytical } \\
\text { performances }\end{array}$} & $\begin{array}{c}\text { Rapid Diagnostic tests } \\
\text { Estimated }(95 \% \mathrm{CI})\end{array}$ & $\begin{array}{c}\text { HBV-Algorithm } \\
\text { Estimated }(95 \% \mathrm{CI})\end{array}$ & $\begin{array}{c}\text { HCV-Algorithm } \\
\text { Estimated }(95 \% \mathrm{CI})\end{array}$ \\
\cline { 2 - 4 } Sample size & $\mathrm{N}=661$ & $\mathrm{~N}=661$ & $\mathrm{~N}=661$ \\
Prevalence & $91.2(88.8,93.2)$ & $50.4(46.6,54.2)$ & $95.6(93.8,96.9)$ \\
Sensibility & $99.0(97.8,99.5)$ & $99.7(98.3,99.9)$ & $98.7(97.5,99.4)$ \\
Specificity & $98.3(90.9,99.7)$ & $98.8(96.9,99.5)$ & $93.1(78.0,98.1)$ \\
PPV & $99.8(99.1,100.0)$ & $98.8(97.0,99.5)$ & $99.7(98.8,99.9)$ \\
NPV & $90.5(80.7,95.6)$ & $99.7(98.3,99.9)$ & $77.14(61.0,87.9)$ \\
PLR & $57.4(8.23,401.0)$ & $81.8(30.9,217.0)$ & $14.3(3.8,54,5)$ \\
NLR & $0.01(0.005,0.02)$ & $0.003(0.0004,0.02)$ & $1.4(0.7,2.7)$ \\
DOR & $4.710^{3}\left(679,45010^{13}\right)$ & $1.4110^{4}\left(3.0610^{3}, 4.5010^{15}\right)$ & $962.6\left(1.9510^{2}, 8.1910^{3}\right)$ \\
Error rate & $1.2(0.5,2.3)$ & $0.8(0.3,1.8)$ & $1.5(0.8,2.8)$ \\
Accuracy & $98.9(97.8,99.5)$ & $99.2(98.2,99.7)$ & $98.5(97.2,99.2)$ \\
Youden J Index & $97.3(93.8,100.0)$ & $98.5(97.2,99.8)$ & $91.8(82.6,100)$ \\
AUC & 98.6 & 99.2 & 95.9 \\
\hline
\end{tabular}

RDTs: Rapid Diagnostic Tests, PPV: Positive Predictive Value, NPV: Negative Predictive Value, PLR: Positive Likelihood Ratio, NLR: Negative Likelihood Ratio, DOR: Diagnostic Odd Ratio, AUC: Area Under Curve. 

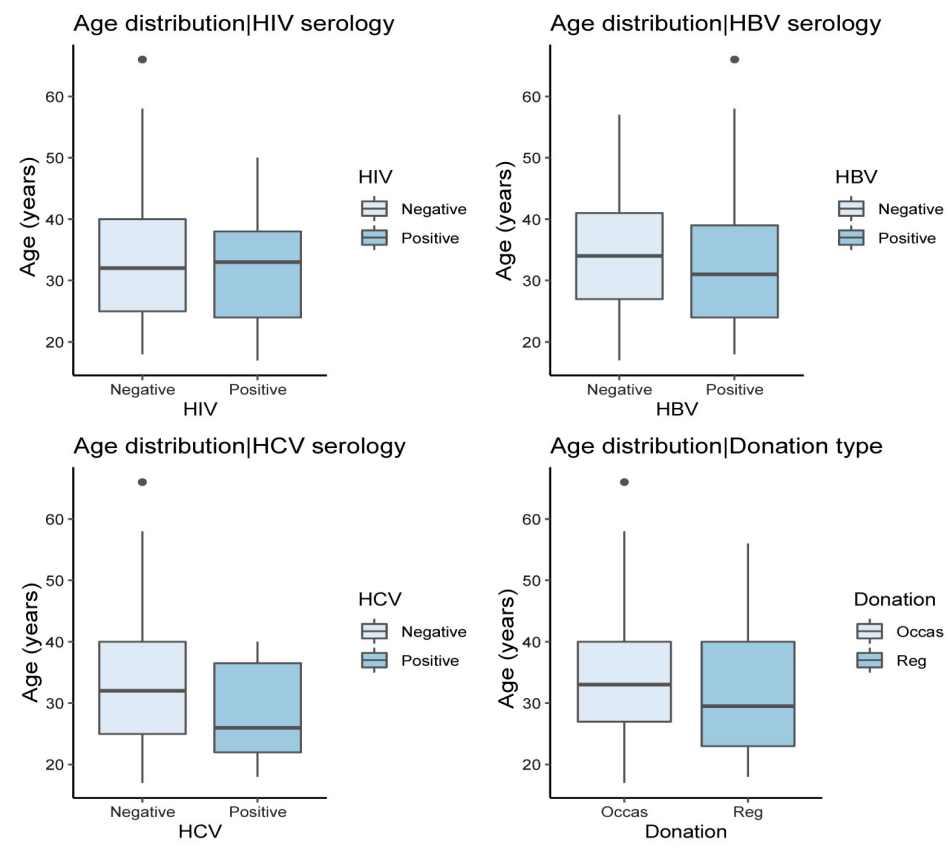

Figure 1. Distribution of median of age according to the serological statute of the tree viral markers and the type of blood donation.

4710. HBV-Ag/Ab RDTs algorithm achieved the following diagnostic accuracy: sens 99.7\% (95\% CI = 98.3, 99.9); spec 98.8\% (95\% CI = 96.9, 95.5); PLR 81.8 $(95 \% \mathrm{CI}=30.9,217.0)$; NLR $0.003(95 \% \mathrm{CI}=0.0004,0.02)$; DOR 14110. The analytical performances of $\mathrm{HCV}-\mathrm{Ab}$ RDTs algorithm were as follow sens $98.7 \%$ (95\% CI = 97.5, 99.4); spec 93.1\% (95\% CI = 78.0, 98.1); PLR 14.3 (95\% CI = 3.8, 54.5); NLR 1.5 (95\% CI = 0.8, 2.8); DOR 962.6 (Table 1). The overall areas under the ROC curves were 98.6\%; 99.2\% and 99.2\%; 95.9\% for HIV-Ag/Ab RDTs, HBV-Ag RDTs and HCV-Ab RDTs algorithms, respectively (Figure 2). The PLR on the nomograms were 57.42; 81.75 and 14.32 for HIV-RDTs, HBV-RDTs and HCV-RDTs, respectively (Figure 2). The parameters evaluating the external validity of RDTs screening for the three viral markers when the theorical prevalence was $<5 \%$ are summarized in Figure 3. At the prevalence $<5 \%$, the Negative Predictive Value (NPV) of the three RDTs were 99.96\%; 99.99\% and 99.94\% for HIV-RDTs, HBV-RDTs and HCV-RDTs, respectively. At the same prevalence we found the following Positive Predictive Values (PPV) 70.82\%, 77.59\% and $37.35 \%$ for the three algorithms, respectively.

\section{Discussion}

As part of the screening of donated blood, RDTs performance should be continuously monitored to identify any variation in analytical performance that may occur upstream of the laboratory due storage conditions, transport or manufacturing defects. Without monitoring, variations in analytical performance could ultimately lead to the misuse, especially in the diagnostic when the prevalence's of viral markers are fairly low. We carried out a cross-sectional study by successive 

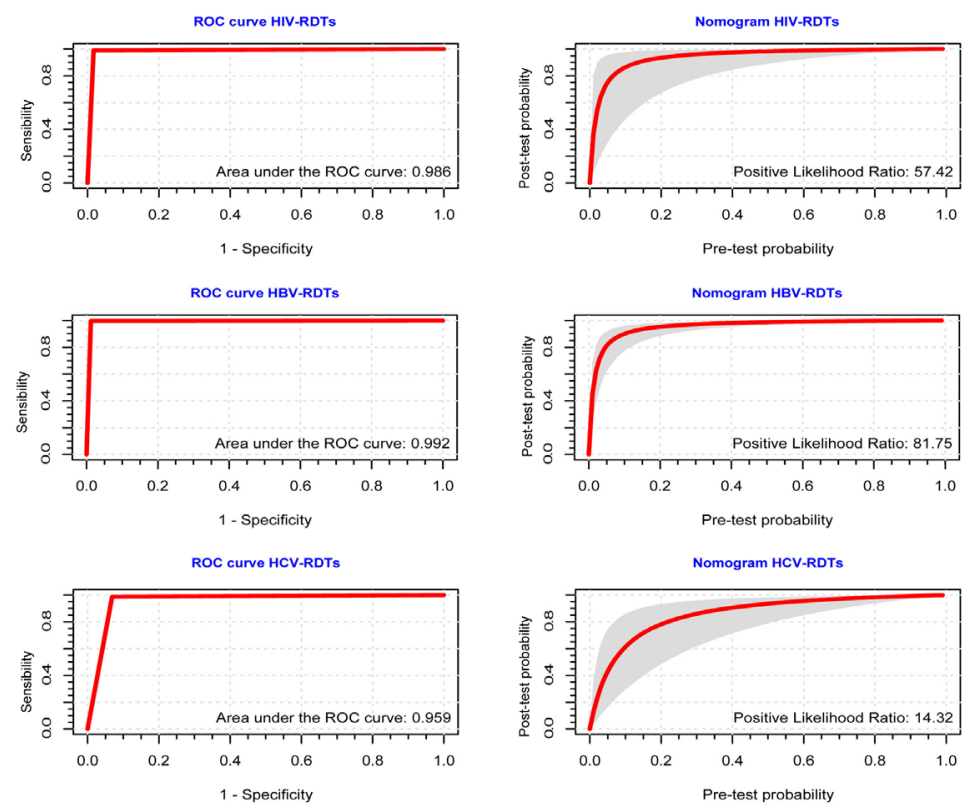

Figure 2. Areas under ROC curve and nomograms of the three algorithms of RDTs.

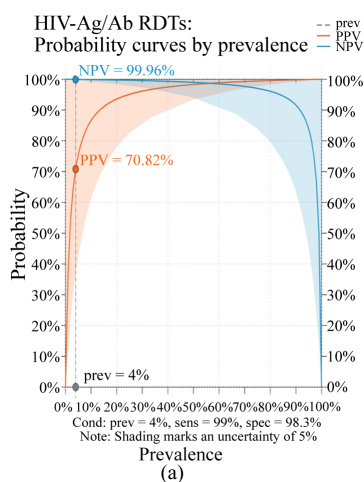

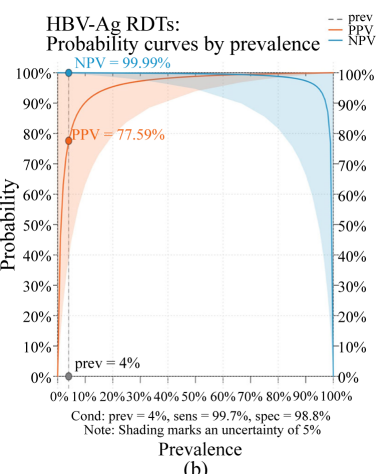

(b)

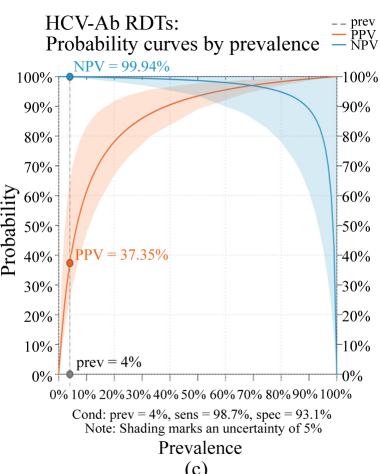

(c)

Figure 3. External validity assessment of RDTs algorithms when theorical prevalence < $5 \%$.

recruitment. Sample size was calculated according to the prevalence of $\mathrm{HBV}-\mathrm{Ag}$ in blood donors according to our previews study Modibo, C., et al. [10]. The performances of the RDTs algorithms were determined by using the ELISA as a reference test. The analytical performances of RDTs algorithms and their 95\% CI were evaluated using the DiagnosisMed package [11]. The ROCR package was used to plot ROC curves and nomograms [12] and the riskyR package was used for external validity of algorithms against theorical prevalence's [13]. Our study recorded $84.0 \%$ of male blood donors with a sex-ratio of 5.2. The age group from 19 to 29 years was the most represented with $37.8 \%$ followed by 30 to 39 years (33.9\%). Our results confirm most of the studies carried out in Mali [10] [14], in Africa [15] and elsewhere [16] which all found a predominance of men and that the donors were predominantly under 40 years of age and are very often family donors. Given that the age distribution according to HIV and HBV serology results and donor type followed a normal law, respecting the assumption of equal- 
ity of variances and donor independence. Thus, we used Student's t-test to compare the means of age according to HIV, HBV serology tests results and donation types. Donors positive to HIV were younger than those negative (32.4 vs 33.4 years). However, this difference was not significant $\mathrm{p}=0.42$. In contrast, donors positive to HBV were significantly younger ( 32.5 vs 34.2 years; $p=0.02$ ) than those who were negative. Likewise, the average age of regular donors was significantly lower than those who were occasional donors (34.0 vs. 32.0 years; $p$ $=0.01)$. As the distribution of age between subjects positive to HCV and those who were negative did not follow the normal law, we used the Mann-Whitney $U$-test for comparison of the median of age. We did not find a significant difference (33.4 vs. 28.7 years; $p=0.22$ ) between the median of ages of HCV positive and negative donors. Overall, our results show that young donors are more likely to have positive serology than older donors. On the other words, the analysis of the donation type shows that regular donors are also younger than occasional donors. This poses a problem of choosing the type of donor which usually should be from the category of regular donors Allain, J.P. et al., 2011 [17]. Regarding the type of donor, occasional donors were significantly more likely to be HBV positive than regular donors $(61.0 \%$ vs $39.0 \% ; \mathrm{p}=0.001)$, respectively. These results are in line with those of Anatole, T., et al., 2009 [18], Moles, S., et al., 2011 [19] who also found that regular donors had low HBV serology positivity compared to occasional donors. However, other authors have reported results that point in the opposite direction Allain, J.P. et al., 2016 [20] and Asenso, M.K. et al., 2014 [21]. The internal validity of our tree algorithms showed a good analytical performances. The diagnostic accuracy for HIV-Ag/Ab RDTs algorithm were as follow: Sensitivity (sens) 99.0\% (95\% CI = 97.8, 99.5); Specificity (spec) 98.3\% (95\% CI = 90.9, 99.7); positive likelihood ratio (PLR) 57.4 (95\% CI = 8.2, 401.0); negative likelihood ratio (NLR) 0.01 ( $95 \% \mathrm{CI}=0.0005,0.02)$; diagnostic odd ratio (DOR) 4710. Our HIV-RDTs algorithm analytical performances are in line with those obtained by Amadou, A., et al., 2005 [22], who reported a sensitivity of $100 \%$ and a specificity of $98.8 \%$. Other authors have reported a fairly low sensitivity; this is the case of Haguiratou, O., et al., 2005 [23] who obtained $58.82 \%$ sensitivity and a specificity of $100 \%$; and Orkuma, J.A., et al., 2014 [24] who reported a sensitivity of $39 \%$ and a specificity of $100 \%$. These good internal validity of our HIV-RDTs algorithm mean that it could be used in the screening of blood donors in transfusion setting. HBV-Ag/Ab RDTs algorithm achieved the following diagnostic accuracy: sens $99.7 \%(95 \% \mathrm{CI}=98.3,99.9)$; spec $98.8 \%$ $(95 \% \mathrm{CI}=96.9,95.5)$; PLR $81.8(95 \% \mathrm{CI}=30.9,217.0)$; NLR $0.003(95 \% \mathrm{CI}=$ $0.0004,0.02$ ) and DOR 14110. Our results are in line with those obtained by Chisenga, C.C., et al., 2018 [25] who reported a PPV of 98.3\%, a NPV of 97.7\%, a sensitivity of $87.9 \%$ and a specificity $99.7 \%$. Bottero, J., et al., 2013 [26] obtained a fairly low sensitivity $80.7 \%$, however, the specificity reported by the same study was comparable, i.e. $99.7 \%$ vs. 98.8 . The internal validity of our HBV-RDTs algorithm also mean that it could be used in the screening of blood donor for 
transfusion setting. The analytical performances of HCV-RDTs algorithm were as follow: sens $98.7 \%(95 \% \mathrm{CI}=97.5,99.4)$; spec $93.1 \%(95 \% \mathrm{CI}=78.0,98.1)$; PLR 14.3 (95\% CI = 3.8, 54.5); NLR 1.5 (95\% CI = 0.8, 2.8); DOR 962.6. Our $\mathrm{HCV}$-algorithm specificity was fairly low than those obtained by Weiming, T., et al., 2017 [27] in a metanalysis study reported a sensitivity and specificity of HCV-RDTS of $98 \%$ and 100\%. Bloch M et al., 2014 [28] in a pilot study in which a total of 12 African countries and 44 laboratories participated reported a mean sensitivity and specificity of $90.1 \%$ and $99.5 \%$ for HCV-RDTs. As for the two previous algorithms, the HCV specificity was fairly low compared to those obtained by other authors such as Bloch, E.M., et al., 2014 [28] and Hess, K.L., et al., 2014 who obtained 99.3\% [29]. Given that in convention the threshold values used to recommend a test for clinical use is NLR $<0.1$ and PLR $\geq 10.0$ Stengel, D., et al., 2003 [30], our HCV-RDTs algorithm do not satisfy these conditions and therefore it could not be recommended for clinical use. To assess the external validity of our algorithms, risky $\mathrm{R}$ package was used to fitted the negative and positive predictive value against the theorical prevalence's of the three viral markers. All of our algorithms achieved a good negative predictive values (NPV) up to $99.9 \%$ at prevalence $<5 \%$ (Figure 3 ) suggesting a high probability in declaring someone negative to the test which quality is required in the screening. However, the positive predictive values (PPV) were very low: 70.8\%, 77.6\% and $37.3 \%$ for HIV, HBV and HCV RDTs algorithms, respectively. These poor PPV suggest that the algorithms have a bad diagnostic value and don't be used in for diagnostic purpose in low prevalences setting. Moreover, at $10 \%$ of prevalence, the PPV were as follow: HIV algorithm (87.8\%), HBV algorithm (91.1\%) and HCV algorithm (63.9\%) whereas the NPV were all up to $99 \%$ for the three algorithms at the same prevalence. At high prevalence (60\%), the PPV were as follow: HIV algorithm (98.9\%), HBV algorithm (99.2\%) and HCV algorithm (95.5\%) whereas the NPV were all up to $97 \%$ for the three algorithms at the same prevalence. Sonia, V.M., et al., 2019 [31] also reported a high NPV but a low PPV when prevalence was $<10 \%$, particularly in HCV-RDTs. In our country, Mali, the prevalence's of three viral markers are $<10 \%$. Therefore our RDTs algorithm are not useful in the diagnostic in this country. Nevertheless, they could be useful where the prevalence's of the viral markers are high, this is the case of East-Africa where HBV prevalence egal $12.01 \%$, HCV prevalence egal 50.91\%; South-Africa where HBV prevalence egal $14.87 \%$ and HIV prevalence egal 49.59\% as reported by Ravendra, P.C., et al., 2020 [7] in a systematic review of important viral diseases in Africa in the light of the "One Health" Concept. Also, they could be useful in diagnostic in the specific populations like prisoners, sex workers and drug abuser who exchange the needle. Univariate analysis did not found no effect of socio-demographic parameters on the three RDTs algorithms. Multivariate analysis also did not show any effect on the HIV algorithm (Figure 4). However, the collection in the mobile cabin: odd ratio 4.2 (95\% CI = $1.6,11.9) ; \mathrm{p}=0.004$ and the positive $\mathrm{HCV}$ coinfection: odd ratio 6.5 (95\% CI = 


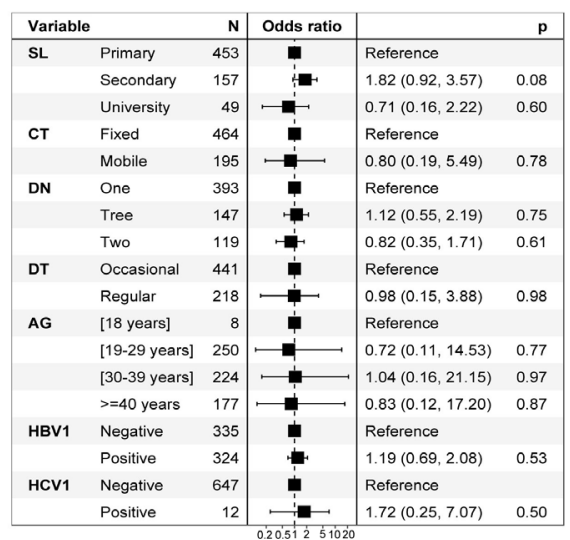

(a)

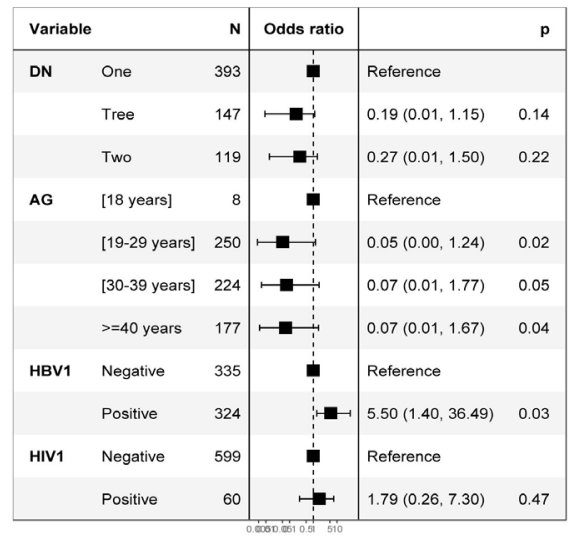

(c)

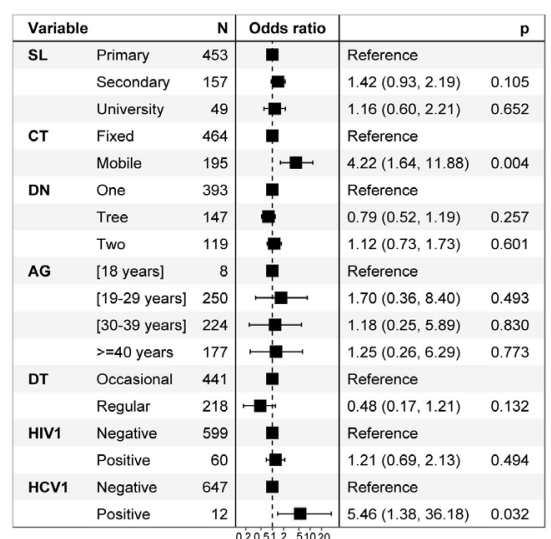

(b)

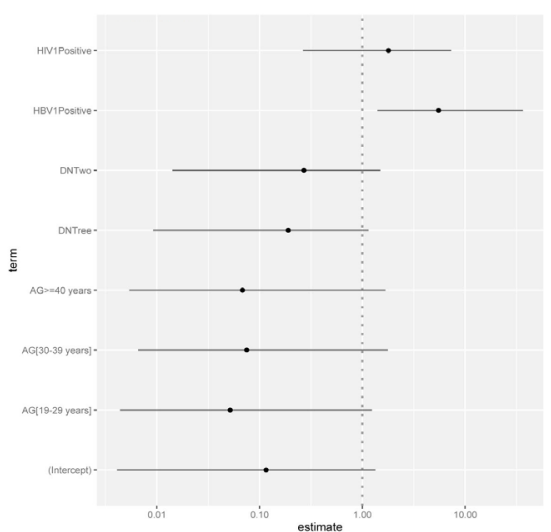

(d)

Figure 4. Multivariate analysis to assess the impact of sociodemographic data on the three algorithms of RDTS. SL = Study level, CT = Collection type, DN = Donation number, DT = Donation type, AG = Age groups. (a) HIV-Ag/Ab; (b) HBV-Ag; (c) HCV-Ab; (d) HCV-Ab.

1.4, 36.2); $\mathrm{p}=0.03$ had a positive effect on the HBV-RDTs algorithm. The age groups (19 - 29 years), (30 - 39 years) and $\geq 40$ years had a slight negative effect on the HCV algorithm whereas HBS coinfection had a positive effect odd ratio $5.6(95 \% \mathrm{CI}=1.4,36.5) ; \mathrm{p}=0.03$ (Figure 4). Logistic regression calls for prudence in the use of the HBV-RDTs and HCV-RDTs algorithms for the screening in the setting of coinfection of HBV and HCV. RDTs algorithms can play a pivotal role in the screening of HIV-Ab/Ag, HBs-Ag in the setting of resources limited-countries where financial and technical expertise shortages are standard fare. However, their use for diagnostic purposes must be done with great caution and the result must necessarily be confirmed with an ELISA or molecular technique particular for HCV-RDTs algorithm which achieved an NLR value $>0.1$.

\section{Conclusion}

RDTs algorithms can play a pivotal role in the screening of HIV-Ab/Ag, HBs-Ag in the setting of resources limited-countries where financial and technical expertise shortages are a standard fare. However, their use for diagnostic purposes must be done with great caution given their poor external validities. The result 
for diagnostic purpose must necessarily be confirmed with an ELISA or molecular technique particularly for HCV-RDTs algorithm which achieved an NLR value $>0.1$. Their use for diagnostic purposes should be reserved in places where the prevalence of viral markers is high.

\section{Acknowledgements}

We are grateful to all study subjects for participating in this study; the staff of Mopti hospital, and the National Center for Transfusion.

\section{Conflicts of Interest}

Authors declare no actual or potential conflict of interest in relation to this article.

\section{Availability of Data and Materials}

The datasets used and/or analyzed during the current study are available from the corresponding author on reasonable request.

\section{References}

[1] World Health Organization (2016) Establishing External Quality Assessment Programmes for Screening of Donated Blood for Transfusion-Transmissible Infections: Implementation Guide. Guidline, Geneva, 80 p.

[2] World Health Organization (2015) HIV Assays: Laboratory Performance and Other Operational Characteristics: Rapid Diagnostic Tests (Combined Detection of HIV-1/2 Antibodies and Discriminatory Detection of HIV-1 and HIV-2 Antibodies): Report 18. Geneva.

[3] DiMinno, G., Perno, C.F., Tiede, A., Navarro, D., Canaro, M., Güertler, L. and Ironside, J.W. (2016) Current Concepts in the Prevention of Pathogen Transmission via Blood/Plasma-Derived Products for Bleeding Disorders. Blood Reviews, 30, 35-48. https://doi.org/10.1016/j.blre.2015.07.004

[4] Bruhn, R., Lelie, N., Busch, M., Kleinman, S. and International NAT Study Group (2015) Relative Efficacy of Nucleic Acid Amplification Testing and Serologic Screening in Preventing Hepatitis C Virus Transmission Risk in Seven International Regions. Transfusion, 55, 1195-1205. https://doi.org/10.1111/trf.13024

[5] An der Heiden, M., Ritter, S., Hamouda, O. and Offergeld, R. (2015) Estimating the Residual Risk for HIV, HCV and HBV in Different Types of Platelet Concentrates in Germany. Vox Sanguinis, 108, 123-130. https://doi.org/10.1111/vox.12204

[6] Pillonel, J., Gallian, P., Sommen, C., Couturier, E., Piquet, Y., Djoudi, R. and Laperche, S. (2014) Assessment of a Transfusion Emergent Risk: The Case of HEV. Transfusion Clinique et Biologique, 21, 162-166. https://doi.org/10.1016/j.tracli.2014.07.004

[7] Ravendra, P., Zelalem, G.D., Ayman, N. and Mohamed, E.Z. (2020) Systematic Review of Important Viral Diseases in Africa in Light of the "One Health" Concept. Pathogens, 9, 301. https://doi.org/10.3390/pathogens9040301

[8] Jorgensen, P., Chanthap, L., Rebueno, A., Tsuyuoka, R. and Bell, D. (2006) Malaria Rapid Diagnostic Tests in Tropical Climates: The Need for a Cool Chain. American Journal of Tropical Medicine and Hygiene, 74, 750-754. 
https://doi.org/10.4269/ajtmh.2006.74.750

[9] Audrey, A., Evan, L., Sheick, O.C., Markos, S., Babacar, F., Mary, L.M., Kadi, O., et al. (2012) Malaria Rapid Diagnostic Test Transport and Storage Conditions in Burkina Faso, Senegal, Ethiopia and the Philippines. Malaria Journal, 11, 406. https://doi.org/10.1186/1475-2875-11-406

[10] Coulibaly, M., Maiga, B., Diawara, M., et al. (2020) Blood Donation Type and Hepatitis B and C Seroepidemiology at Hôpital Sominé DOLO de Mopti. GSC Biological and Pharmaceutical Sciences, 13, 25-31. https://doi.org/10.30574/gscbps.2020.13.3.0393

[11] Brasil, P. (2020) DiagnosisMed: Diagnostic Test Accuracy Evaluation for Medical Professionals. Computer Software (R Package Version 0.2.3). http://CRAN.R-project.org/src/contrib/Archive/DiagnosisMed

[12] Sing, T., Sander, O., Beerenwinkel, N. and Lengauer, T. (2005) ROCR: Visualizing Classifier Performance in R. Bioinformatics, 21, 3940-3941. https://doi.org/10.1093/bioinformatics/bti623

[13] Neth, H., Gaisbauer, F., Gradwohl, N. and Gaissmaier, W. (2018) Riskyr: A Toolbox for Rendering Risk Literacy More Transparent. Social Psychology and Decision Sciences, University of Konstanz, Germany. Computer Software (R Package Version 0.2.0, Dec. 20, 2018). https://CRAN.R-project.org/package=riskyr

[14] Hamadi, T., et al. (2013) Etude comparative de le seroprevalence des marqueurs $\mathrm{VIH}, \mathrm{VHB}$ et VHC des dons de sang en collecte fixe et mobile à BAMAKO. Thèse de Pharmacie, Bamako, No P-48.

[15] Ngama, K.C., Mukuku, O., Matanda, S.K., Manika, M.M., Kyabu, V.K., Kasamba, E.I., Mawaw, P.M., Mwamba, C.M. and Kapend, L. (2016) Seroprevalence and Epidemiological Profile of Blood Donors at the Lubumbashi University Clinics, Democratic Republic of Congo. The Pan African Medical Journal, 23, 175.

[16] Pillonel, J., Legrand, D., Sommen, C. and Laperche, S. (2012) Surveillance épidémiologique des donneurs de sang et risque résiduel de transmission du VIH, de l'HTLV, du VHC et du VHB par transfusion en France entre 2008 et 2010. BEH, 39, 438-442.

https://www.santepubliquefrance.fr/maladies-et-traumatismes/infections-sexuellem ent-transmissibles/vih-sida/documents/article/surveillance-epidemiologique-des-do nneurs-de-sang-et-risque-residuel-de-transmission-du-vih-de-l-htlv-du-vhc-et-duvhb-par-transfusion-en-france

[17] Allain, J.P. (2011) Moving on from Voluntary Non-Remunerated Donors: Who Is the Best Blood Donor? British Journal of Haematology, 154, 763-769.

https://doi.org/10.1111/j.1365-2141.2011.08708.x

[18] Tounkara, A., Diakite, M., Noumsi, G.T., Sarro, Y.D., Siddiqui, S. and Parta, M. (2009) Retrospective Surveillance of HIV Prevalence in Blood Donors Can Help in the Selection of the Best Social Group for Blood Donation in Mali. Transfusion Medicine, 19, 252-259. https://doi.org/10.1111/j.1365-3148.2009.00950.x

[19] Mole, S., Onana, E. and Biholong, D. (2011) VIH et facteurs de risque chez les donneurs de sang de remplacement familiaux et les bénévoles à l'hôpital central de Yaoundé, Cameroun. Bulletin de la Société de Pathologie Exotique, 104, 226-231. https://doi.org/10.1007/s13149-011-0163-3

[20] Allain, J.P. and Sibinga, C.T. (2014) Family Donors Are Critical and Legitimate in Developing Countries. Asian Journal of Transfusion Science, 10, 5-11.

https://doi.org/10.4103/0973-6247.164270 
[21] Asenso-Mensah, K., Achina, G., Appiah, R., Owusu-Ofori, S. and Allain, J.P. (2014) Can Family or Replacement Blood Donors Become Regular Volunteer Donors? Transfusion, 54, 797-804. https://doi.org/10.1111/trf.12216

[22] Amadou, A., Kouka, N., Mahamane, A.E. and Chanteau, S. (2005) Evaluation de cinq tests rapides et de deux algorithmes pour le diagnostic de l'infection par le VIH au Niger. Bulletin de la Société de Pathologie Exotique, 98, 5-8. https://pubmed.ncbi.nlm.nih.gov/15915963

[23] Ouedraogo, H., et al. (2005) Evaluation des performances de sept tests de dépistage du VIH utilisés au CNTS de Bamako. Thèse de Pharmacie, Bamako, N05P18.

[24] Orkuma, J.A., Egesie, J.O., Banwat, E.B., Ejele, A.O., Orkuma, J.H. and Bako, I.A. (2014) HIV Screening in Blood Donors: Rapid Diagnostic Test versus Enhanced ELISA. Nigerian Journal of Medicine, 23, 192-200.

https://pubmed.ncbi.nlm.nih.gov/25185375

[25] Chisenga, C.C., Musukuma, K., Chilengi, R., Zürcher, S., Munamunungu, V., Siyunda, A., Ojok, D., Bauer, S., Wandeler, G. and Vinikoor, M. (2018) Field Performance of the Determine HBsAg Point-of-Care Test for Diagnosis of Hepatitis B Virus Co-Infection among HIV Patients in Zambia. Journal of Clinical Virology, 98, 5-7. https://doi.org/10.1016/j.jcv.2017.11.005

[26] Bottero, J., Boyd, A., Gozlan, J., Lemoine, M., Carrat, F., Collignon, A., Boo, N., Dhotte, P., Varsat, B., Muller, G., Cha, O., Picard, O., Nau, J., Campa, P., Silbermann, B., Bary, M., Girard, P.M. and Lacombe, K. (2013) Performance of Rapid Tests for Detection of HBsAg and Anti-HBsAb in a Large Cohort, France. Journal of Hepatology, 58, 473-478. https://doi.org/10.1016/j.jhep.2012.11.016

[27] Tang, W.M., Chen, W., Amini, A., Boeras, D., Falconer, J., Kelly, H., Peeling, R., Varsaneux, O., Tucker, J.D. and Easterbrook, P. (2017) Diagnostic Accuracy of Tests to Detect Hepatitis C Antibody: A Meta-Analysis and Review of the Literature. BMC Infectious Diseases, 17, 695. https://doi.org/10.1186/s12879-017-2773-2

[28] Bloch, E.M., Shah, A., Kaidarova, Z., Laperche, S., Lefrere, J.J., van Hasselt, J., et al. (2014) A Pilot External Quality Assurance Study of Transfusion Screening for HIV, HCV and HBs Ag in 12 African Countries. Vox Sanguinis, 107, 333-342. https://doi.org/10.1111/vox.12182

[29] Hess, K.L., Fisher, D.G. and Reynolds, G.L. (2014) Sensitivity and Specificity of Point-of-Care Rapid Combination Syphilis-HIV-HCV Tests. PLoS ONE, 9, e112190. https://doi.org/10.1371/journal.pone.0112190

[30] Stenge, D., Bauwens, K., Sehouli, J., Ekkernkamp, A. and Porzsolt, F. (2003) A Likelihood Ratio Approach to Meta-Analysis of Diagnostic Studies. Journal of Medical Screening, 10, 47-51. https://doi.org/10.1258/096914103321610806

[31] Vázquez-Morón, S., Ardizone Jiménez, B., Jiménez-Sousa, M.A., et al. (2019) Evaluation of the Diagnostic Accuracy of Laboratory-Based Screening for Hepatitis C in Dried Blood Spot Samples: A Systematic Review and Meta-Analysis. Scientific Reports, 9, Article No. 7316. https://doi.org/10.1038/s41598-019-41139-8 